\title{
Effect of Homogeneous Condensation on the Interaction of Supersonic Moist Air Jets with Resonance Tube
}

\author{
M. M. Ashraful Alam ${ }^{1 \dagger}$, S. Matsuo ${ }^{2}$ and T. Setoguchi ${ }^{3}$ \\ ${ }^{1}$ Department. of Mechanical Engineering, Khulna University of Engineering \& Technology, Khulna-9203, \\ Bangladesh \\ ${ }^{2}$ Department of Advanced Technology Fusion, Saga University, 1, Honjo-machi, Saga-shi, Saga 840-8502, Japan \\ ${ }^{3}$ Institute of Ocean Energy, Saga University, 1, Honjo-machi, Saga-shi, Saga 840-8502, Japan
}

†Corresponding Author Emai: rana94.kuet@gmail.com

(Received April 18, 2011; accepted November 6, 2011)

\begin{abstract}
The Hartmann tube, can use for flow-control, is a device which generates high intensity sound through the shock wave oscillations, are created by the interaction of the supersonic jet. In this study, two-phase flow simulations are carried out to characterize the effect of non-equilibrium condensation on the unsteady flowfield of the Hartmann resonance tube. This present numerical work provides a new insight on the flow dynamics and acoustics of the resonance tube - including the shock nature, the tube gas heating, and the effect of non-equilibrium condensation on the flow structure. A TVD numerical method is applied to the Reynolds and Favre-averaged Navier-Stokes equations, and droplet growth equation of liquid phase production. The simulations are performed over a range of nozzle pressure ratios. The numerically simulated flow structure of under-expanded supersonic jets is compared with experimental data. Moreover, the predicted frequency of end wall pressure fluctuations is compared with the experimental results.
\end{abstract}

Keywords: Compressible flow, Condensation, Non-equilibrium, Shock oscillations, Two-phase

$\begin{array}{ll} & \\ d & \text { diameter of the nozzle, } \mathrm{m} \\ D & \text { diameter of the resonance tube, } \mathrm{m} \\ D_{\mathrm{e}} & \text { nozzle exit diameter, } \mathrm{m} \\ D_{\mathrm{m}} & \text { diameter of the Mach disk, } \mathrm{m} \\ g & \text { condensate mass fraction, }- \\ I & \left.\text { nucleation rate, } 1 / \mathrm{m}^{3} \cdot \mathrm{s}\right) \\ \kappa & \text { Boltzmann constant, } \mathrm{J} / \mathrm{K} \\ k & \text { turbulent kinetic energy, } \mathrm{m}^{2} / \mathrm{s}^{2} \\ L & \text { tube length, } \mathrm{m} \\ L_{\mathrm{m}} & \text { location of the Mach disk, } \mathrm{m} \\ M & \text { Mach number, - } \\ p & \text { static pressure, Pa } \\ \rho & \text { density, } \mathrm{kg} / \mathrm{m}^{3} \\ r & \text { droplet radius, } \mathrm{m} \\ r_{\mathrm{c}} & \text { critical droplet radius, } \mathrm{m} \\ R & \text { eddy viscosity, Pa.s }\end{array}$

\section{NOMENCLATURE}

Re Reynolds number, -

$R_{\mathrm{T}} \quad$ turbulent Reynolds number, -

$\sigma \quad$ surface tension, $\mathrm{N} / \mathrm{m}$

$S \quad$ initial degree of supersaturation, -

$t \quad$ time, s or temperature, ${ }^{\circ} \mathrm{C}$

$T$ temperature, $\mathrm{K}$

$\mu \quad$ dynamic viscosity, $\mathrm{Pa} \cdot \mathrm{s}$

$\tau \quad$ instantaneous time, $\mathrm{s}$

0 stagnation

$b \quad$ ambient

$l \quad$ laminar quantity

$l \quad$ liquid

$m \quad$ mixture or Mach disk

$v \quad$ vapor

\section{INTRODUCTION}

In recent, investigating the unsteady flow phenomena of resonance tubes have become a strongly motivated research field by their potential application as high frequency actuators for suppressing high speed jet noise and supersonic flow oscillations in open cavities. Hartmann (1931) observed that high frequency pressure fluctuations are generated when an air jet oscillator such as the resonance tube is facing an under expanding jet from a nozzle. He reported that these high frequency

pressure fluctuations are strongly dependent on the length of the tube and its distance from the nozzle exit. Hartmann explained that the tube should be placed in the compression region termed as 'zones of instability' to obtain high frequency tones. Sprenger (1954) made an in-depth study of the effects of nozzle-tube spacing, nozzle stagnation pressure, and tube length on the endwall temperature (Kawahashi et al. 1979). He observed large variations in end-wall temperature with slight changes in nozzle pressure ratio or spacing between nozzle and tube. The high temperatures and intense 
heating obtained at the resonance tube end wall due to high dissipation were studied by Rakowsky et al. (1974) for application as explosive igniter in rocket engines and scramjets (Hamed et al. 2003).

Brocher et al. (1969) explained the flow oscillation mechanism in terms of compression and evacuation phases as the jet penetrates into the tube and compresses the air, then the compressed air is expunged out of the tube into the atmosphere (Samimy et al. 2002). They carried out experiments for jet Mach numbers ranging between 0.1 and 2.0 placing a thin cylindrical body at the nozzle center in order to achieve evacuation of the tube in the expansion phase. Sarohia and Back (1979) discuss briefly, three possible modes of operation from their experimental investigations of unsteady flows in a Hartmann tube, depending upon the tube, gap size, and jet Mach number. They investigated the effect of jet Mach number on resonance tube operation and predicted the switching from jet regurgitant to jet screech mode with increased jet Mach number.

Stanek et al. (2001) demonstrated that Hartmann resonance tube based fluidic actuators are more effective than other passive devices for noise suppression in high speed flow over cavities. Cain et al. (2002) conducted a numerical investigation to study the effect of pressure ratio and free-stream flow on the pressure oscillations in a resonance tube. Raman et al. (2004) conducted an experimental study to obtain the desirable frequency in high bandwidth resonance tube actuator though the variation of gap and tube length. Kastner and Samimy (2002) studied the effect of tube length, jet Mach number and separation distance on the power spectra of far acoustic field and the near pressure field. Hamed et al. (2002) conducted a numerical investigation of the unsteady flow in a resonance tube with a supersonic jet to characterize the pressure oscillations and the high amplitude low frequency tones generated by the tube in the jet regurgitant mode. In a subsequent study Hamed et al. (2003) also studied the jet Mach number effect on the resonance tube flow.

The supersonic moist air jets have not yet received the same level of attention as dry air jet interaction. In these cases, the non-equilibrium condensation may occur in the jet expansion region, and the surrounding gas will be heated by the release of latent heat of condensation. From Otobe et al. (2006), the supersonic jet flows with non-equilibrium condensation is quite different from that without condensation. They observed that the occurrence of the non-equilibrium condensation in the supersonic jet reduced the intensity pressure fluctuation in the flow field. However, so far, the effect of nonequilibrium condensation on the unsteady flows in the resonance tube resulting from the impingement of supersonic moist air jet onto the tube has not been investigated till now. Therefore, it requires performing investigation on the flow characteristics especially the unsteady complex shock structure in the part of the resonance tube flow for better understanding the fundamentals of tube gas heating, flow structure and flowfield oscillations when the under-expanded supersonic moist air jets impinge onto the resonance tube.

The principal thrust of the present computational work is to acquire an in-depth understanding of the unsteady resonance tube flow, the fundamentals of tube gas heating and flowfield oscillations. The simulations are performed over a range of nozzle pressure ratios resulting in different jet Mach numbers. Moreover, we focus on the effect of non-equilibrium condensation on that unsteady resonance tube flow characteristics.

\section{COMPUTATIONAL ANALYSIS}

\subsection{Governing Equations}

Computational predictions were performed by using two-dimensional, unsteady, compressible NavierStokes equations, that combine a nucleation rate equation and a droplet growth equation (Hill 1966, Sislian 1975, Adam 1996, Matsuo et al. 2004) of condensate nuclei. The present computations neglected interphase velocity and temperature slip, and they were discussed in detail by Matsuo et al. (2004). It was assumed that the condensate particles have a negligible effect on the pressure field. The conservation equations of mass, momentum and energy for the viscous adiabatic compressible flow of the mixture are similar to their single phase flow. The resulting governing equations are given in the axisymmetric coordinate system $(x, r)$ ( $r$ : the radial distance from the axis),

$$
\begin{aligned}
\frac{\partial \boldsymbol{U}}{\partial t}+\frac{\partial \boldsymbol{E}}{\partial x}+\frac{\partial \boldsymbol{F}}{\partial r}= & \frac{1}{\operatorname{Re}}\left(\frac{\partial \boldsymbol{R}}{\partial x}+\frac{\partial \boldsymbol{S}}{\partial r}\right)+\frac{1}{r} \boldsymbol{H}_{1} \\
& +\boldsymbol{H}_{2}+\boldsymbol{Q}
\end{aligned}
$$

where $U$ is the vector of conservative variables, $E$ and $F$ are inviscid flux vectors, and $R$ and $S$ are viscous flux vectors. $H_{1}, H_{2}$, and $Q$ are the source terms of axisymmetry, turbulence and condensation, respectively. Once a droplet is able to form by the homogeneous nucleation, its further growth is determined by the droplet growth law as follows (Sislian 1975):

$$
\begin{aligned}
\frac{d g}{d t}= & 4 \pi \rho_{l}\left[\frac{I(t)}{\rho_{m}(t)} \cdot \frac{r_{c}^{3}(t)}{3}\right. \\
& +\frac{d r}{d t} \int_{t_{i}}^{t}\left\{\frac{I(\tau)}{\rho_{m}(\tau)}\left(r_{c}(\tau)+\int_{\tau}^{t} \frac{d r}{d t} d \theta\right)^{2}\right\} d \tau
\end{aligned}
$$

In the above equation, $g$ and $t$ is condensate mass fraction and time, respectively. $\rho$ is density. $r_{\mathrm{c}}$ and $I$ are critical droplet radius and nucleation rate per unit time and volume, respectively. Subscripts $m$ and $l$ refer to mixture and liquid, respectively.

In the present study, homogeneous nucleation of moist air was modeled by the Frankel's empirical equation, formulated based on the classical nucleation theory, for nucleation rate $I$ as follows (Sislian 1975):

$I=\frac{1}{\rho_{l}}\left(\frac{2 m_{v} \sigma}{\pi}\right)^{1 / 2}\left(\frac{p_{v}}{\kappa T}\right)^{2} \exp \left(-\frac{4 \pi r_{c}^{2} \sigma}{3 \kappa T}\right)$

where $\kappa, \sigma$ and $p_{v}$ are Boltzmann constant, surface tension and vapor pressure of condensate droplet, respectively. In the present simulation, $r_{\mathrm{c}}, d r / d t, \rho_{l}$, surface tension, saturation vapor pressure of condensate 
droplet of radius $r$, latent heat and other coefficients were given on referring Sislian (1975) and Adam (1996). Validation of the condensation model using these equations and quantities was shown in Refs. (Matsuo et al. 2003, 2004, Alam et al. 2008, 2010).

To close the governing equations, a modified $k-R$ model (Goldberg 1994, 1996; Heiler 1999) was employed to solve turbulence stresses in the present computations. The turbulent kinetic energy $k$ and the undamped eddy viscosity $R$ are determined by the following transport equations.

$$
\begin{gathered}
\frac{\partial\left(\rho_{m} k\right)}{\partial t}+\frac{\partial\left(\rho_{m} k u\right)}{\partial x}+\frac{\partial\left(\rho_{m} k v\right)}{\partial y}=\frac{\partial}{\partial x}\left[\left(\mu_{l}+\frac{\mu_{t}}{\sigma_{k}}\right) \frac{\partial k}{\partial x}\right] \\
+\frac{\partial}{\partial y}\left[\left(\mu_{l}+\frac{\mu_{t}}{\sigma_{k}}\right) \frac{\partial k}{\partial y}\right]+P_{k}-\frac{\left(\rho_{m} k\right)^{2}}{\rho_{m} R} \\
\frac{\partial\left(\rho_{m} R\right)}{\partial t}+\frac{\partial\left(\rho_{m} R u\right)}{\partial x}+\frac{\partial\left(\rho_{m} R v\right)}{\partial y}=\frac{\partial}{\partial x}\left[\left(\mu_{l}+\frac{\mu_{t}}{\sigma_{k}}\right) \frac{\partial R}{\partial x}\right] \\
+\frac{\partial}{\partial y}\left[\left(\mu_{l}+\frac{\mu_{t}}{\sigma_{k}}\right) \frac{\partial R}{\partial y}\right]-\frac{\rho_{m} C_{\mu}}{\sigma_{R}}\left[\left(\frac{\partial R}{\partial x}\right)^{2}+\left(\frac{\partial R}{\partial y}\right)^{2}\right] \\
+\left(2-C_{R 1}\right) \frac{R}{k} P_{k}-\left(2-C_{R 2}\right) \rho_{m} k
\end{gathered}
$$

where $P_{k}$ is the turbulent production modeled in terms of the Boussinesq concept:

$P_{\mathrm{k}}=\left[\mu_{t}\left(\frac{\partial u_{i}}{\partial x_{i}}+\frac{\partial u_{j}}{\partial x_{i}}-\frac{2}{3} \frac{\partial u_{k}}{\partial x_{k}} \delta_{i j}\right)-\frac{2}{3} \rho_{m} k \delta_{i j}\right] \frac{\partial u_{i}}{\partial x_{j}}$

The undamped eddy viscosity $R$ (Adam 1996) is given by

$R \equiv \frac{k^{2}}{\varepsilon}$

Here $k$ is the turbulent kinetic energy and $\varepsilon$ is its dissipation. In the above equation, $u_{i}$ are the Cartesian mean velocity components; $x_{i}$ are the corresponding coordinates; $\mu_{l}$ and $\mu_{t}$ are the molecular and eddy viscosities, respectively.

In the present simulation, we have introduced a limiter function (Yamamoto et al. 1991) to suppress the instability in the region of Mach disk as

$P_{k}=\min \left[P_{k}, C_{2} \cdot C_{b} \cdot \rho_{m} \cdot \varepsilon \cdot \operatorname{Re} / C_{1}\right]$

where $C_{\mathrm{b}}$ is a limiting parameter determined by the empirical approach and

$C_{1}=1.44, C_{2}=1.92 \times\left[1-0.3 \exp \left(-R_{T}^{2}\right)\right], C_{b}=1.001$

$C_{\mu}=0.09, \sigma_{k}=1.0, \sigma_{R}=1.080, C_{R 1}=1.44, C_{R 2}=1.92$

The eddy viscosity is given by

$\mu_{t}=C_{\mu} \cdot f_{\mu} \cdot \rho_{m} \cdot R$

where the near-wall damping function $f_{\mu}$ is given as

$f_{\mu}=\frac{\tanh \left(\alpha \cdot R_{T}\right)}{\tanh \left(\beta \cdot R_{T}\right)}$

where
$R_{T}=\frac{\rho_{m} k^{2}}{\mu_{l} \varepsilon}=\frac{\rho_{m} R}{\mu_{l}}, \quad \alpha=0.05, \quad \beta=\frac{C_{\mu}^{3 / 4}}{\kappa^{k}}$

\subsection{Numerical Methods}

The governing equation systems were nondimensionalized with the reference values at the inlet conditions upstream of the nozzle, and then these were mapped from the physical plane into a computational plane of a general transform. A third-order TVD (total variation diminishing) finite difference scheme with MUSCL (Yee 1989) approach was used to discretize the spatial derivatives of the governing equations. The TVD scheme is based on a concept aimed at preventing the generation of new extreme in the flow solution. The principal conditions for a TVD scheme are that maxima must be non-increasing, minima non-decreasing, and no new local extreme may be created. Thus, a discretization methodology with TVD properties allows it to resolve a shock wave without any spurious oscillations of the solution. The MUSCL interpolation has to be enhanced by the so-called limiter function or limiter, if the flow region contains strong gradients. The purpose of the limiter is to suppress non-physical oscillation of the solution. A second-order central difference scheme was applied to discretize the viscous terms. For time integration, a fractional step method which consisted in splitting the discretized space operators into products of one-dimensional operators was used.

$U^{n+1}=L_{r}\left(\frac{\Delta t}{2}\right) \cdot L_{x}(\Delta t) \cdot L_{r}\left(\frac{\Delta t}{2}\right) U^{n}$

Here, $L_{x}$ and $L_{r}$ are the split operators in $x$ and $r$ directions, respectively. $n$ and $n+1$ are the successive states at time step of $\Delta t$, respectively.

\subsection{Computational Conditions}

Axisymmetric supersonic impinging moist air jet flows driven by the cylindrical straight nozzle with exit diameter of $\phi d=20.3 \mathrm{~mm}$ (characteristic length) interacting with the resonance tube were considered in the present computation. The nozzle is composed of a convergent curved entrance wall that has a radius of curvature $R=10.15 \mathrm{~mm}$ and following straight wall with a length of $0.4 R$. The schematic view of the computational domain and boundary conditions used in the present study is shown in Fig.1. The gap, $s$ between the nozzle exit and tube inlet was kept constant at $2 d$. The ratio of tube diameter to nozzle exit diameter $D / d=1.25$ was kept constant throughout the computation. The tube length $L$ to diameter $D$ ratio $(L / D)$ is equal to 3 . A deflector shield of outer radius of $12 D$ was placed at the entrance of the resonance tube to prevent the working gas from flowing over the outside of the tube.

The current simulations were conducted for axisymmetric two-phase flows. Moist air was assumed as the working gas, and its initial relative humidity at the nozzle supply is given by a function of the initial degree of super saturation $S_{0}$. The jet pressure ratio of 
moist air was defined as the ratio of total pressure $p_{0}$ at the nozzle supply to back pressure $p_{\mathrm{b}}\left(=p_{0} / p_{\mathrm{b}}\right)$. In the present study, $p_{0} / p_{\mathrm{b}}$ was varied from 2.6 to 8.27 , and the initial degree of super saturation $S_{0}$ of moist air was changed between 0 (dry air) and 0.8 . The upstream total temperature $T_{0}$ and total pressure $p_{0}$ were assumed to be maintained constant at $298.15 \mathrm{~K}$ and $101.3 \mathrm{kPa}$, respectively, through the whole computations.

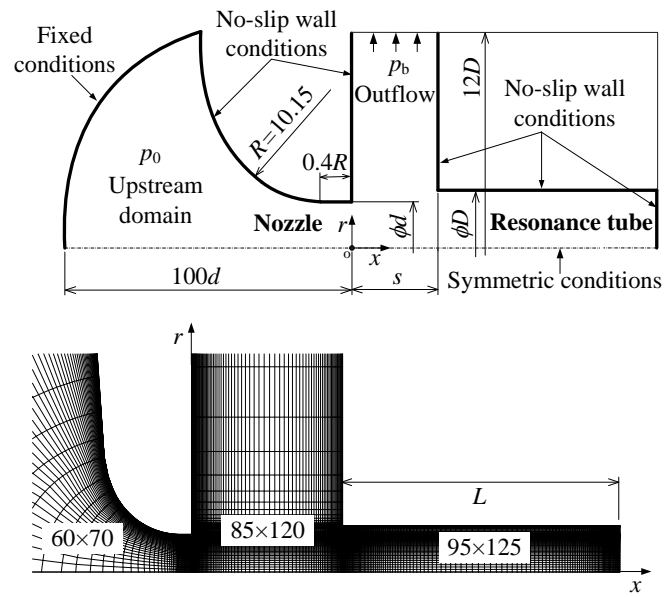

Fig. 1. Schematic view of computational domain (up) and typical grid system (down)

Fixed boundary conditions were applied to the upstream boundary of the computational domain and the downstream boundary was subject to the outflow conditions. Adiabatic and no-slip boundary conditions were applied to the solid wall surfaces. Further, condensate mass fraction was assumed to be zero at the solid wall surfaces. The axisymmetric condition was used at the boundary of the nozzle center line.

According to several preliminary computations, it was known that numerical waves were propagating far upstream of the nozzle inlet. Hence, the upstream domain in the present computations extended to the distance of $100 d$ upstream from the nozzle exit, while the downstream domain covered the region of $50 \mathrm{~d}$, in the $r$-direction. This wide computational domain is required for domain independent solution. A typical grid system employed in the present computations is shown in Fig. 1. The structured grids were clustered in the flow regions with large gradient such as the wall boundary layer, shock wave and nozzle exit. The computational grids closest to the nozzle walls are located at $0.0004 d$ away from the walls. Several preliminary computations have been performed on different computational grid meshes to investigate the grid-independent solutions. According to these results, the number of grids applied is $60 \times 70$ in the nozzle region, $85 \times 120$ in the jet plume region and $125 \times 95$ in the tube.

\section{RESUlt AND Discussion}

\subsection{Comparison with Experimental Results}

The validation of the present computational code in predicting the effect of non-equilibrium condensation on the unsteady resonance tube flow characteristics was investigated through the experimental and numerical study of under-expanded supersonic moist air jets. In both experimental and numerical studies, underexpanded supersonic jet flows were driven by the cylindrical straight nozzle with exit diameters of $\phi D_{\mathrm{e}}=$ $12.7 \mathrm{~mm}$. The nozzle is composed of a convergent curved entrance wall which has a radius of curvature $R=$ $D_{\mathrm{e}}$ and following straight wall with a length of $0.4 D_{\mathrm{e}}$. The schematic of computational domain and boundary conditions in simulating the under-expanded supersonic jets is shown in Fig. 2. The atmospheric air as the working gas contains more or less moisture, depending on the daily weather conditions. In the present experiment, the condition of $S_{0}=0$ which corresponds to the dry air was never achieved due to limited capacity of the dehumidification system employed. The lowest value of $S_{0}$ in the experiment was at most 0.15 . Thus, in the present study, the condition of $S_{0}=0.15$ was assumed to correspond to that of the dry air in computations. The range of pressure ratio $p_{0} / p_{\mathrm{b}}$ was varied between 3.8 and 6.2. In computations, the initial degree of supersaturation $S_{0}$ was changed only in the range from 0 (dry air) to 0.7 . The upstream total temperature $T_{0}$ and total pressure $p_{0}$ in the numerical study were the same as the experimental study, and values of $T_{0}$ and $p_{0}$ are $298.15 \mathrm{~K}$ and $101.3 \mathrm{kPa}$, respectively.

Figurs. 3 and 4 show comparisons of the computed and experimental under-expanded supersonic jets under different pressure ratios and $S_{0}$. The upper halves in these figures are the computed iso-density contours and the lower halves are the shadowgraph pictures. For the dry air of $S_{0}=0$, the jet is slightly under-expanded (see Fig. $3(\mathrm{a})$ ), and the resulting weak shock waves have the regular reflection at the point ' $\mathrm{P}$ '. However, at $S_{0}=0.7$, the shock system transforms into the Mach reflection, as shown in Fig. 3(b). The difference in two jets structures at same pressure ratio is stimulated by the released latent heat due to the occurrence of non-equilibrium condensation of moisture component. On the contrary, in case of $p_{0} / p_{\mathrm{b}}=6.2$ in Fig. 4(a), the dry air jet is strongly under-expanded at the nozzle exit, compared with Fig. 3(a), and barrel shocks are formed due to the differences in pressure between the under-expanded gas and ambient gas. Therefore, these shocks reflect near the jet axis. Consequently, the Mach disk is formed near the jet axis. At the interaction of the Mach disk with barrel shock, a triple-point is formed. In the downstream of the triple point the slip line is observed. However, it is again found that the non-equilibrium condensation appreciably influences the shock structure of the moist air jet, as observed in Fig. 4(b); it is likely that the diameter of the Mach disk grows with $S_{0}$. In addition, as the pressure ratio increases, the shock cells extend streamwise that result in an increase in the length of 'supersonic core' of jets. The predicted iso-density contours, in all cases, are nearly the same as the results obtained by the xperiments, and it is mentioned that the present computations predict the under-expanded dry and moist air jets with good accuracy.

The quantitative comparisons of the predicted and experimental data for the location $L_{\mathrm{m}} / D_{\mathrm{e}}$ and diameter $D_{\mathrm{m}} / D_{\mathrm{e}}$ of Mach disk of under-expanded supersonic jets at $p_{0} / p_{\mathrm{b}}=6.2$ is presented in Table 1 . Here, location of Mach disk is measured from the nozzle exit, and both the location and diameter of the Mach disk are 
normalized by the nozzle exit diameter $D_{\mathrm{e}}$. Table 1 shows that values of location and diameter of the Mach disk predicted in the present computation are in very good agreement with the experimental data.

Another validation work has been conducted through the comparison between the predicted results of unsteady flow behavior of resonance tube interacting with the supersonic jets and experimental data of Sarohia and Back (1979). The frequencies of endwall pressure fluctuations under different operating condition $\left(p_{0} / p_{\mathrm{b}}\right)$ are measured, and compared with the experimental data as represented graphically in Fig. 5. The present results are found to be in good agreement with those of Sarohia and Back (1979).

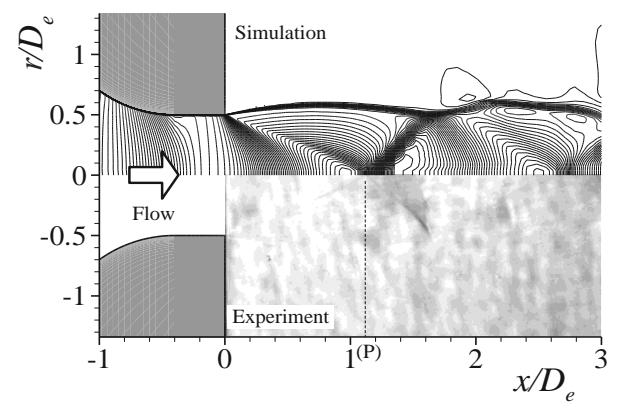

(a) $S_{0}=0$

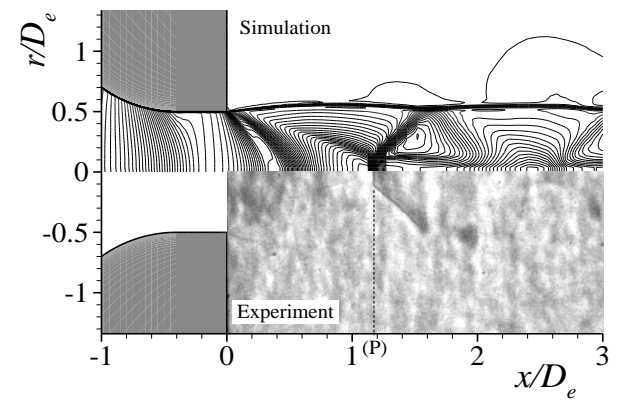

(b) $S_{0}=0.7$

Fig. 3. Comparisons of the computed and experimental under-expanded supersonic jets at $p_{0} / p_{\mathrm{b}}=3.8$

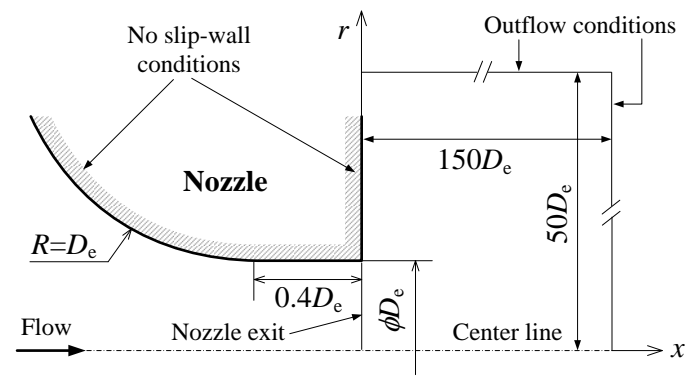

Fig. 2. Computational domain of under-expanded supersonic jets

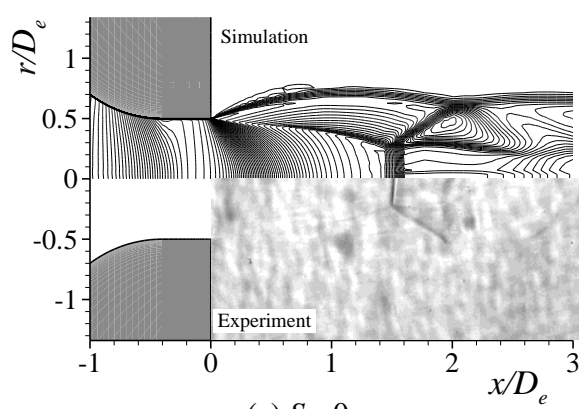

(a) $S_{0}=0$

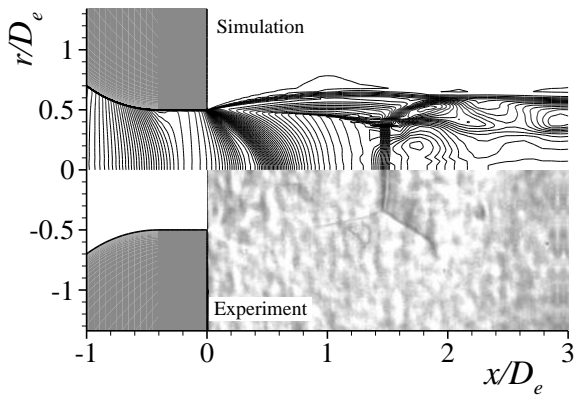

(b) $S_{0}=0.7$

Fig. 4. Comparisons of the computed and experimental under-expanded supersonic jets at $p_{0} / p_{\mathrm{b}}=6.2$ 


\subsection{Effect of Non-equilibrium Condensation}

To characterize the oscillatory behavior of unsteady resonance tube flow the pressure fluctuations at tube endwall was measured and presented graphically in Fig. 6; while the power spectra of pressure oscillation at tube endwall were evaluated using FFT analysis

Table 1 Comparison of predicted location and diameter of Mach disk of under-expanded supersonic moist air jets with the present experimental data $\left(p_{0} / p_{\mathrm{b}}=6.2\right)$

\begin{tabular}{|l|c|c|c|c|}
\hline \multirow{2}{*}{} & \multicolumn{2}{|c|}{$S_{0}=0$} & \multicolumn{2}{c|}{$S_{0}=0.7$} \\
\cline { 2 - 5 } & $L_{\mathrm{m}} / D_{\mathrm{e}}$ & $D_{\mathrm{m}} / D_{\mathrm{e}}$ & $L_{\mathrm{m}} / D_{\mathrm{e}}$ & $D_{\mathrm{m}} / D_{\mathrm{e}}$ \\
\hline Computation & 1.526 & 0.533 & 1.551 & 0.640 \\
\hline Experiment & 1.545 & 0.521 & 1.564 & 0.646 \\
\hline
\end{tabular}

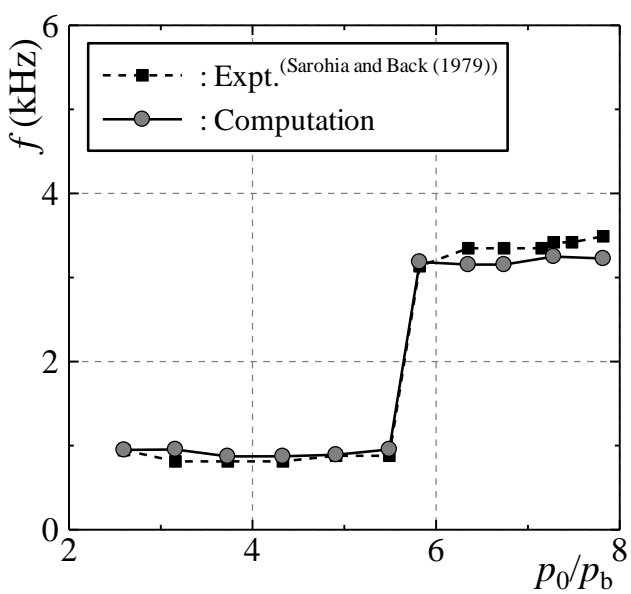

Fig. 5. Frequencies of endwall pressure fluctuations

and demonstrated in Fig. 7. Figure 6 shows typical pressure fluctuations at tube endwall for $p_{0} / p_{\mathrm{b}}=2.6$, $3.16,6.35$ and 8.27. The end wall pressure traces for $p_{0} / p_{\mathrm{b}}=2.6$ and $3.16\left(S_{0}=0\right)$ in Figs. 6(a) and (b) show oscillations at the fundamental resonance frequency is about $950 \mathrm{~Hz}$. On the contrary, the amplitude of pressure oscillations at higher nozzle pressure ratio (for $p_{0} / p_{\mathrm{b}}=6.35$ and $\left.8.27\left(S_{0}=0\right)\right)$ is much lower, as shown in Figs. 6(c) and (d). This is also in agreement with the experimental observation of Sarohia and Back (1979). Again, from Fig. 6, amplitudes of static pressure of moist air jets at $S_{0}=0.6$ and 0.8 (Figs. 6(a-d)) become small compared with those of dry air jets $S_{0}=0$. However, from Fig. 7, peaks of the power spectrum density are reduced in case with non-equilibrium condensation. Moreover, peaks of the power spectrum density are reduced with an increase in the initial degree of supersaturation, $S_{0}$. On the contrary, at higher nozzle pressure ratio (for $p_{0} / p_{\mathrm{b}}=6.35$ and 8.27) the frequency of endwall pressure fluctuations for $S_{0}=0.6$ and 0.8 is lowered at about $955 \mathrm{~Hz}$, as shown in Figs. 7(c) and $7(\mathrm{~d})$.

Figures 8 (a) and 8(b) show static pressure distributions along tube axis during one period of flowfield oscillations for cases of $p_{0} / p_{\mathrm{b}}=2.6$ and 6.35 , respectively
$\left(S_{0}=0\right)$. From Fig. 8(a), pressure distributions during one cycle of flowfield oscillation show that there exist shock waves in the first shock cell of jet and just in front of the tube inlet. At the inflow phases, compression waves are generated and propagate towards the tube endwall. Consequently, the rise in endwall gas temperature, which occurred through these trains of compression waves, is nearly isentropic; showed about $430 \mathrm{~K}$. Therefore, at the outflow phase,

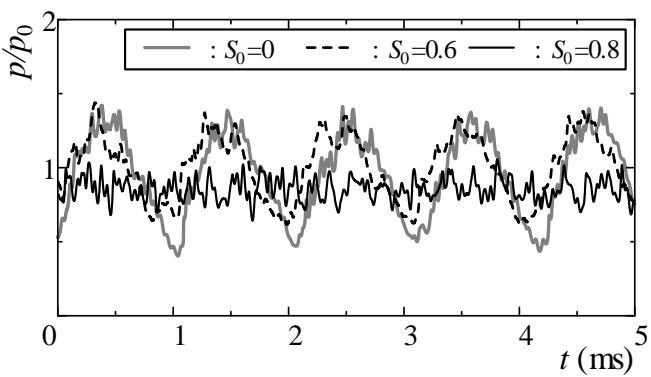

(a) $p_{0} / p_{\mathrm{b}}=2.6$

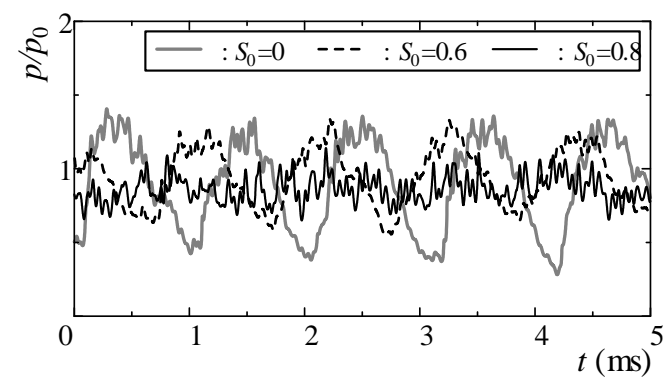

(b) $p_{0} / p_{\mathrm{b}}=3.16$

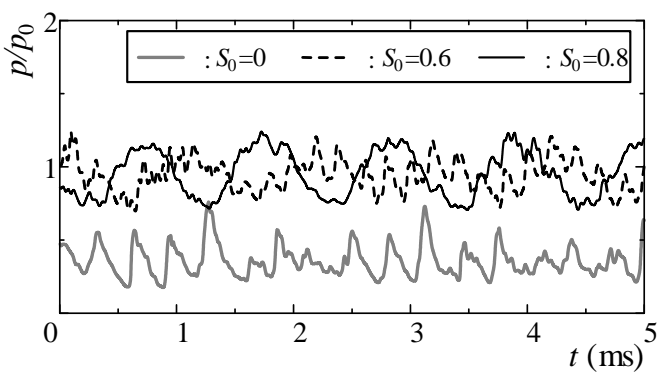

(c) $p_{0} / p_{\mathrm{b}}=6.35$

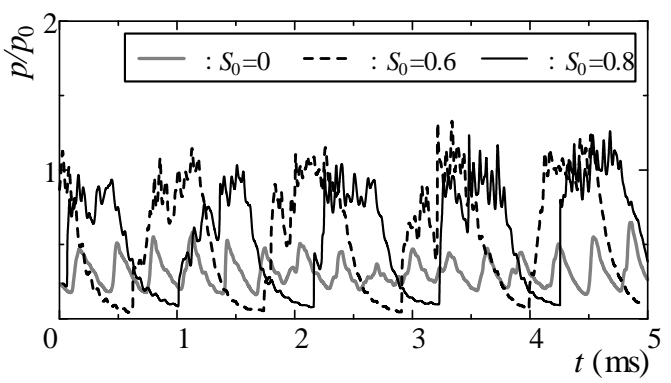

(d) $p_{0} / p_{\mathrm{b}}=8.27$

Fig. 6. Time history of static pressure at tube endwall

the endwall tube gas is cooled back to the initial temperature isentropically. At higher nozzle pressure 
ratio $\left(p_{0} / p_{\mathrm{b}}=6.35\right)$, a strong shock wave (Mach disk) is present in the first shock cell, as shown in Fig. 8(b). Thus, weak compression waves are generated, and create a feedback loop of flow in the tube.

Figures 9(a) and 9(b) illustrate typical contours of isodensity and pressure for the cases of $p_{0} / p_{\mathrm{b}}=2.6$ and 6.35 , respectively. At a small nozzle pressure ratio $\left(p_{0} / p_{\mathrm{b}}=2.6\right)$ in Fig. $9(\mathrm{a})$, the dry air jet $\left(S_{0}=0\right)$ is weakly

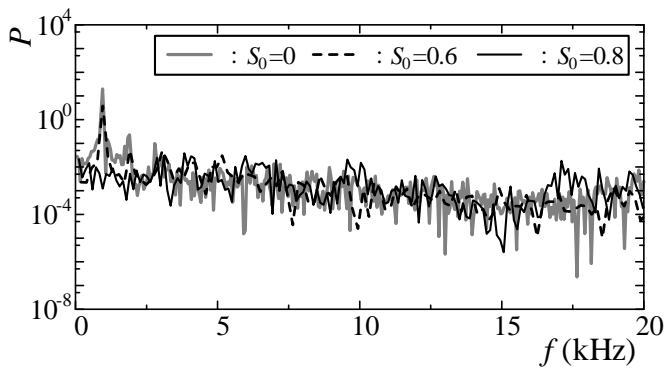

(a) $p_{0} / p_{\mathrm{b}}=2.6$

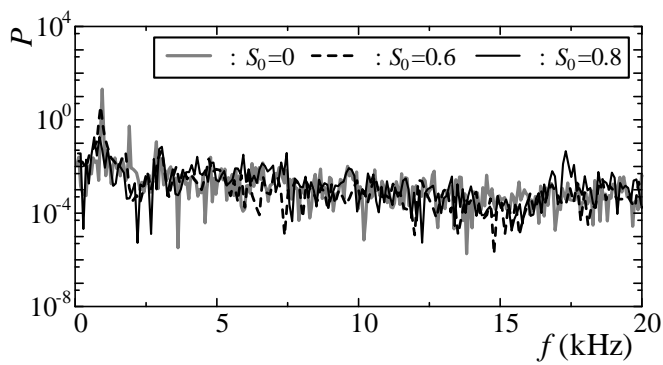

(b) $p_{0} / p_{\mathrm{b}}=3.16$

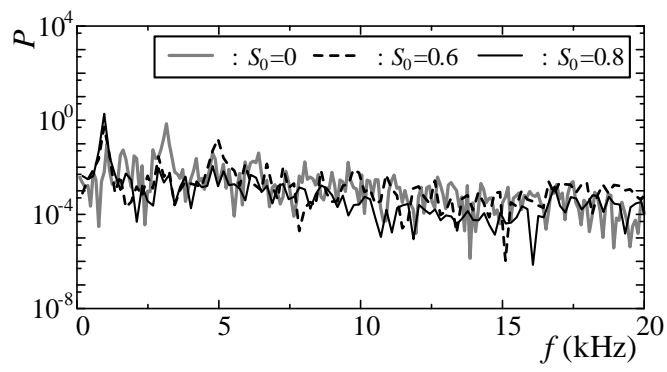

(c) $p_{0} / p_{\mathrm{b}}=6.35$

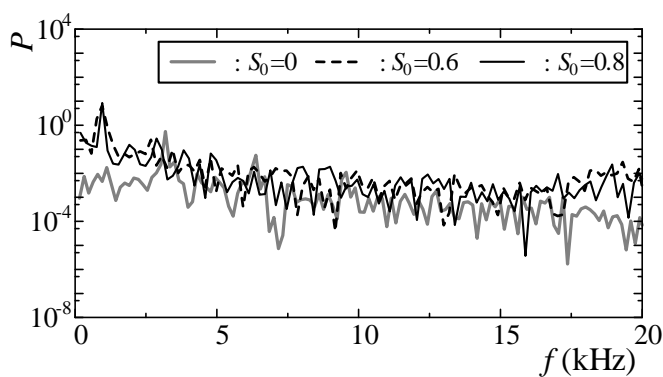

(d) $p_{0} / p_{\mathrm{b}}=8.27$

Fig. 7. Distribution of power spectra of pressure oscillation at tube endwall

under-expanded, and resulting weak shock waves have regular reflection in the flowfield. On the contrary, in case of $p_{0} / p_{\mathrm{b}}=6.35$ in Fig. 9(b), the dry air jet is strongly under-expanded at nozzle exit, and results in the formation of Mach disk near the jet axis. From some of

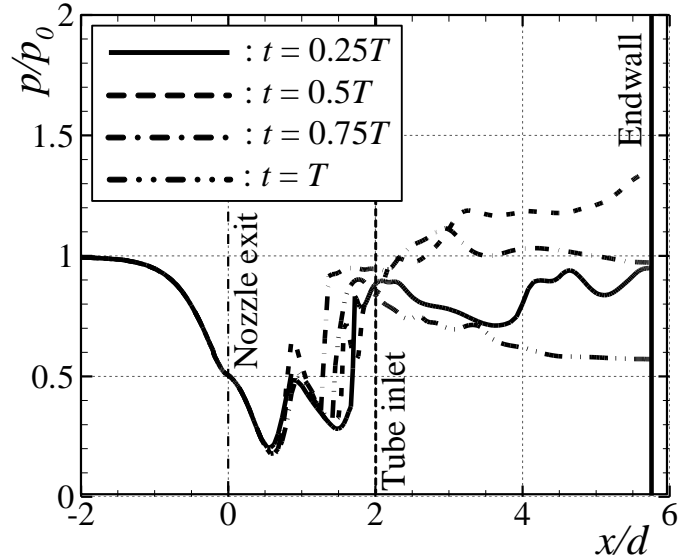

(a) $p_{0} / p_{\mathrm{b}}=2.6$

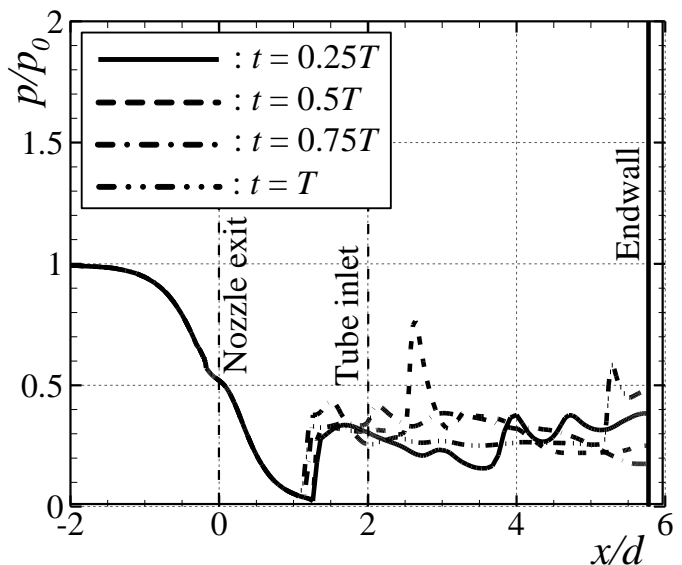

(b) $p_{0} / p_{\mathrm{b}}=6.35$

Fig. 8. Variation of distributions of static pressure along tube axis $\left(S_{0}=0\right)$

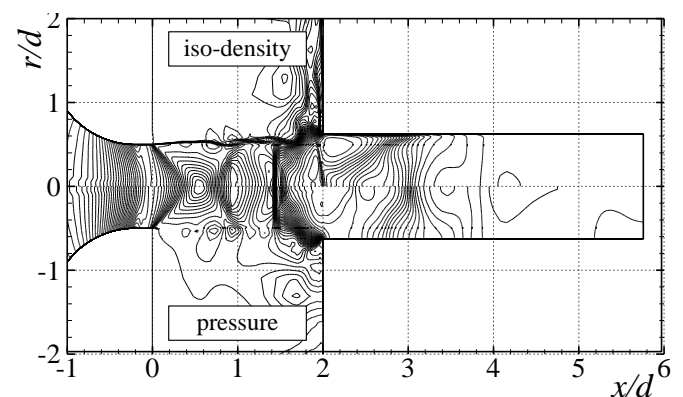

(a) $p_{0} / p_{\mathrm{b}}=2.6$

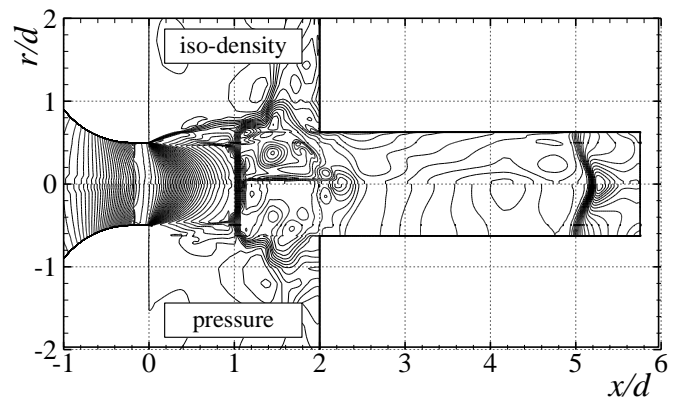

(b) $p_{0} / p_{\mathrm{b}}=6.35$

Fig. 9. Typical iso-density and pressure contours $\left(S_{0}=0\right)$ 
our studies, it can be mentioned that the jet flowfield can be exhibited Mach reflection in the first shock cell at pressure ratio $p_{0} / p_{\mathrm{b}}$ beyond 3.8 (Alam et al. 2010; Otobe et al. 2007).

Variations of distribution of static pressure $p$, condensate mass fraction $g$ and nucleation rate $I$ on the tube axis during one period of flowfield oscillation for cases of $p_{0} / p_{\mathrm{b}}=2.6\left(S_{0}=0.6\right)$ and $p_{0} / p_{\mathrm{b}}=6.35\left(S_{0}=0.8\right)$ are shown in Figs. 10(a) and 10(b), respectively. As seen from Fig. 10(a), the pressure amplitudes during flowfield oscillation become smaller compared to that of without the non-equilibrium condensation (Fig. 8(a)), and there is no significant strength of compression waves marching through the tube. The nucleation rate begins to increase from upstream of the nozzle exit and reaches the maximum at onset of condensation. Moreover, the condensate mass fraction begins to increase at the onset of condensation and distributed over the tube. In addition, for cases of $p_{0} / p_{\mathrm{b}}=2.6$, $S_{0}=0.8 ; p_{0} / p_{\mathrm{b}}=3.16, S_{0}=0.6$ and 0.8 , the variation of static pressure, condensate mass fraction and nucleation rate are almost similar to those in case of $p_{0} / p_{\mathrm{b}}=2.6$, $S_{0}=0.6$ presented in Fig. 10(a). In both cases of $p_{0} / p_{\mathrm{b}}=2.6$ and 3.16 , the amplitude of static pressure during oscillation for $S_{0}=0.8$ are reduced compared to

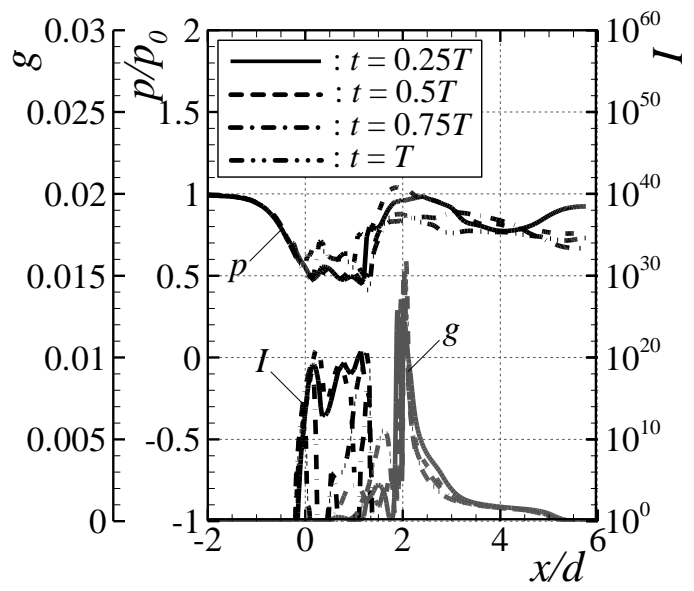

(a) $p_{0} / p_{\mathrm{b}}=2.6, S_{0}=0.6$

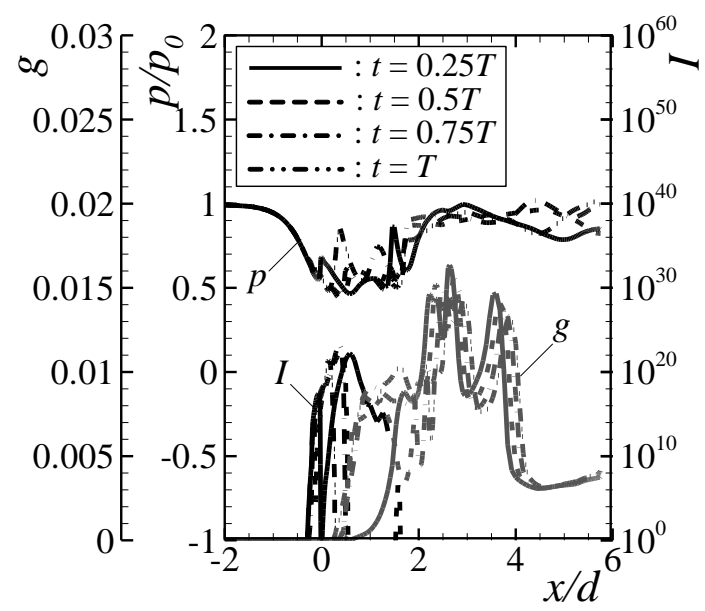

(b) $p_{0} / p_{\mathrm{b}}=6.35, S_{0}=0.8$

Fig. 10. Variation of distributions of static pressure, condensate mass fraction and nucleation rate along tube axis

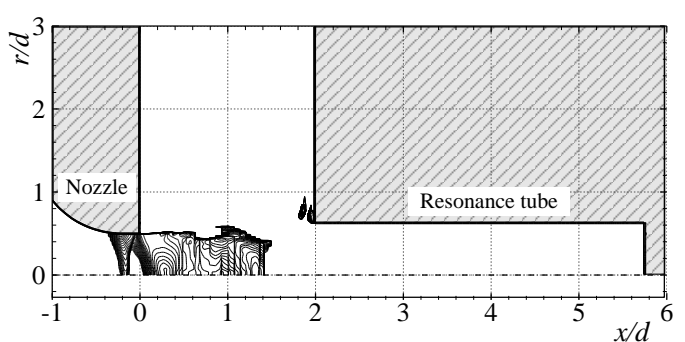

(a) $p_{0} / p_{\mathrm{b}}=2.6, S_{0}=0.6$

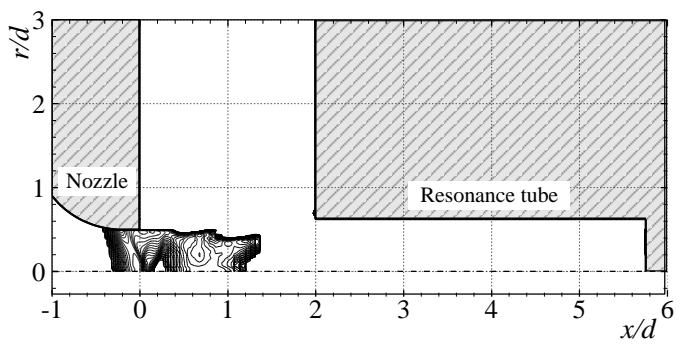

(b) $p_{0} / p_{\mathrm{b}}=6.35, S_{0}=0.8$

Fig. 11. Typical contours of nucleation rate

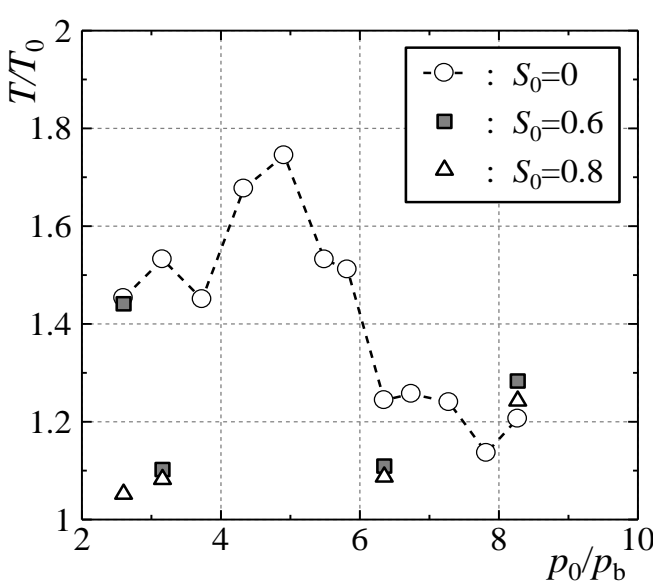

Fig. 12. Relation between nozzle pressure ratio and end wall gas temperature

that of case for $S_{0}=0.6$.

On the contrary, at higher nozzle pressure ratio $\left(p_{0} / p_{\mathrm{b}}=6.35, S_{0}=0.8\right)$ in Fig. 10(b) the generation of condensate nuclei in the upstream region of the nozzle exit, and the rapid increase of condensate mass fraction in the jet expansion fan reduces the average maximum jet Mach number by approximately $60 \%$. The distributions of static pressure shows that due to the reduction of average maximum jet Mach number the flowfield behaves similarly like the resonance tube flow for $p_{0} / p_{\mathrm{b}}=2.6, S_{0}=0$. In addition, the variation of static pressure, condensate mass fraction and nucleation rate for cases of $p_{0} / p_{\mathrm{b}}=6.35, S_{0}=0.6 ; p_{0} / p_{\mathrm{b}}=8.27, S_{0}=0.6$ and 0.8 are almost similar to those in case of $p_{0} / p_{\mathrm{b}}=6.35$, $S_{0}=0.8$.

Figure 11(a) and (b) show typical contours of nucleation rate $I$ for cases of $p_{0} / p_{\mathrm{b}}=2.6, S_{0}=0.6$ and 
$p_{0} / p_{\mathrm{b}}=6.35, S_{0}=0.8$, respectively. From Figs. 11(a) and (b), the instantaneous contours of nucleation rate exhibit that the condensate nuclei begin to generate from the region upstream of the nozzle exit, and distribute through the jet expansion region. It is mentioned that the condensate mass fraction begins to increase rapidly at the onset of condensation and distributed over the region downstream of the nozzle exit and expansion fan.

The effect of nozzle pressure ratio $\left(p_{0} / p_{\mathrm{b}}\right)$ on the endwall gas temperature of $L / D=3.0$ resonance tube with nozzle-to-tube spacing $s / d=2.0$ is shown in Fig. 12. The endwall temperature reaches the maximum at a nozzle pressure ratio about 5.0. As nozzle pressure ratio is increased further, the gas temperature decreased, and reaches almost to the ambient temperature due to the formation of relatively weak waves inside the tube. Moreover, the occurrence of non-equilibrium condensation reduces the endwall gas temperature in cases of $S_{0}=0.6$ and 0.8 .

\section{COnClusion}

The present study dealt with the computational work to investigate the effect of non-equilibrium homogeneous condensation on the interaction of under-expanded supersonic moist air jets with resonance tube. The axisymmetric, unsteady, compressible Navier-Stokes equations system with coupled droplet growth equation are used to investigate the oscillatory features of endwall pressure and structural changes in the resonance tube flow when under-expanded supersonic moist air jets impinge onto the tube. Furthermore, the numerical code used in the present work has been validated through the comparison of predicted results with experimental data. The results obtained are summarized as follows: the occurrence of nonequilibrium condensation in the jet flowfield at a lower nozzle pressure ratio reduces the amplitude of pressure oscillation. Peaks of the power spectrum density are reduced in case with non-equilibrium condensation. On the contrary, at a higher nozzle pressure ratio, the endwall pressure fluctuates at a lower frequency due to the effect of non-equilibrium condensation. The occurrence of non-equilibrium condensation reduces the endwall temperature rise.

\section{REFERENCES}

Adam, S. (1996). Numerische und Experimentelle untersuchung Instationärer Düsenströmungen mit Energiezufuhr durch Homogene Kondensation. Ph.D. Dissetation, Fakultät für Maschinenbau, Universität Karlsruhe (TH), Germany.

Alam, M.M.A., Matsuo, S. and Setoguchi, T. (2008). Characteristics of supersonic impinging moist air jets. International Journal of Turbo and Jet Engines 25, 221-231.
Alam, M.M.A., Matsuo, S. and Setoguchi, T. (2010). Effect of non-equilibrium homogeneous condensation on the self-induced flow oscillation of supersonic impinging jets. International Journal of Thermal Sciences 49, 2078-2092.

Cain, A., Kerschen, E., Raman, G. and Khanafseh, S. (2002). Simulation of powered resonance tubes: Effects of pressure ratio and free-stream flow. AIAA Paper 2002-2821.

Goldberg, U.C. (1994). Toward a pointwise turbulence model for wall-bounded and free shear flows. Transaction of ASME 116, 72-76.

Goldberg, U.C. (1996). Exploring a three-equation $R-k$ $\varepsilon$ turbulence model. Journal of Fluids Engineering $118,795-799$.

Hamed, A., Das, K. and Basu, D. (2002). Numerical simulation of unsteady flow in resonance tube. AIAA Paper 2002-1118.

Hamed, A., Das, K. and Basu, D. (2003). Numerical simulation and parametric study of HartmannSprenger tube based powered device. AIAA paper 2003-0550.

Heiler, M. (1999). Instationäre Phänomene in Homogen/ Heterogen Kondensierenden Düsen- und Turbinenströmungen. Ph. D. Dissertation, Fakultät für Maschinenbau, Universität Karlsruhe (TH), Germany.

Hill, P.G. (1966). Condensation of water vapor during supersonic expansion in nozzles. Journal Fluid Mechanics 25, 593-620.

Kastner, J. and Samimy, M. (2002). Development and characterization of Hartmann tube base fluidic actuators for high speed flow control. AIAA Paper 2002-0128.

Kawahashi, M. and Suzuki, M. (1979). Generative mechanism of air column oscillations in a Hartmann-Sprenger tube excited by an air jet issuing from a convergent nozzle. Journal of Applied Mathematics and Physics 30(6), 797-810.

Matsuo, S. Otobe, Y. Tanaka, M. Kashimura, H., Setoguchi, T. and Yu, S. (2004). Effect of nonequilibrium condensation on axisymmetric underexpanded jet. International Journal of Turbo and Jet Engines 21, 193-201. 
M.M. Ashraful Alam et al. / JAFM, Vol. 6, No. 2, pp. 203-212, 2013.

Matsuo, S., Tanaka, M., Setoguchi, T. and Kaneko, K. (2003). Effect of non-equilibrium condensation of moist air on flow fields in Ludwieg tube (without condensation in upstream of the nozzle). Transaction of Japanese Society of Mechanical Engineering 69, 1163-1170 (in Japanese).

Otobe, Y., Matsuo, S., Tanaka, M., Kashimura, H. and Setoguchi, T. (2007). A study on characteristics of under-expanded condensing jet. JSME International Journal, Series B, 49(4), 1165-1172.

Raman, G., Khanafseh, S., Cain, A. and Kerschen, E. (2004). Development of high bandwidth powered resonance tube actuators with feedback control. Journal of Sound and Vibration 269, 1031-1062.

Samimy, M., Kastner, J. and Debiasi, M. (2002). Control of a high-speed impinging jet using a Hartmann-Tube based fluidic actuator. AIAA paper 2002-2822.

Sarohia, V. and Back, L.H. (1979). Experimental investigation of flow and heating in a resonance tube. Journal of Fluid mechanics, 94(4), 649-672.
Sibulkin, M. (1963). Experimental investigation of energy dissipation in a resonance tube. Zeitschrift für Angewandte Mathematik und Physik (ZAMP) 14(6), 695-703.

Sislian, J.P. (1975). Condensation of water vapor with or without a carrier gas in a shock tube. UTIAS Report 201.

Stanek, M.J., Raman, G., Kibens, V., Ross, J.A., Odedra, J. and Peto, J.W. (2001). Suppression of cavity resonance using high frequency forcing The characteristic signature of effective devices. AIAA Paper 2001-2128.

Yamamoto, S. and Daiguji, H. (1998). Unsteady Navier-Stokes simulation of turbulent flows through a supersonic compressor cascade. Proceedings of the Symposium, ASME and JSME Joint Fluids Engineering Conference, 1st, Portland, OR; USA, 73-79.

Yee, H.C. (1989). A class of high-resolution explicit and implicit shock capturing methods. NASA TM89464. 